\title{
Interictal EEG changes in patients with seizure disorder: experience in Bangladesh
}

\author{
Rajib Nayan Chowdhury, ATM Hasibul Hasan*, Kazi Mohibur Rahman, Badrul Alam Mondol, Sudip Ranjan Deb \\ and Quazi Deen Mohammad
}

\begin{abstract}
The objective of this study was to determine the changes and sensitivity of electro encephalogram during interictal period and to evaluate the finding in the clinically suspected seizure events in a tertiary care hospital of Bangladesh. This cross sectional study was carried out in the Electrophysiology Laboratory of Dhaka Medical College Hospital from July 2010 to July 2011, which included 767 patients. EEG was obtained through scalp electrodes following international 10/20 system. Patient and their attendants were interviewed using a semi structured questionnaire. The EEG findings and clinical seizure events were then compared. Among the 767 epilepsy patients most were children (39.9\% less than 10 years old) and young adult (33.2\% in 11-20 years age group). Female patients predominantly had seizure than male ( $57 \%$ and $43 \%$ respectively). The overall sensitivity of EEG in yielding abnormal interictal epileptiform discharges was $62.7 \%$. About $48.5 \%$ of them were diagnosed as localization related epilepsy and $11.7 \%$ were generalized epilepsy. Morphology showed spike and wave in $74 \%$ and sharp and wave in $11 \%$ tracings. Only $2 \%$ had slow waves. The presence of an interictal spike/sharp wave helps to confirm a clinical diagnosis of epilepsy, aids in defining the epilepsy syndrome, provides information that assists in planning drug management.
\end{abstract}

Keywords: Interictal EEG

\section{Introduction}

Though Gibbs and his colleagues (Gibbs et al. 1935) discovered the pattern of epileptic discharges in 1935, the first description of an epileptic crisis dates back to $3000 \mathrm{~b}$. c. Since the introduction, electroencephalogram (EEG) has been used to diagnose and manage epilepsy. A seizure is any clinical event caused by abnormal electrical discharge in the brain, whilst epilepsy is tendency to have recurrent seizure (Allen et al. 2010). In other words the term 'Epilepsy' refers to recurrent and unprovoked seizures. There is wide variation of incidence of epilepsy worldwide due to variation in classification system of epilepsy and methodology adopted in different studies (Yacubian 2000). The life time incidence of epilepsy varies from 2\% to 5\% (WHO report on Epilepsy in South East Asia). With the incidence of 2-10 per thousand for south East Asian countries, it is estimated that there are 1.5-2 million people suffering from epilepsy in Bangladesh (WHO report on Epilepsy in South East Asia). Marino et

\footnotetext{
* Correspondence: parag007us@gmail.com

Dhaka Medical College Hospital, Dhaka, Bangladesh
}

al (1986) showed that the incidence is highest at both extreme of ages, especially in neonatal period and after $6^{\text {th }}$ decade. Even with the tremendous advances in neurodiagnostic procedures, the role of EEG is not abolished. The interictal spike/sharp remains the hallmark of epilepsy, by demonstrating the cortical hyperexcitability and hypersynchrony, which may persists in the "normal" interictal state. The aim of our study was to determine the changes and sensitivity of interictal EEG in demonstrating the pathological abnormality and evaluate the presence of paroxysm of epileptiform discharges with clinically suspected seizure events. Other technologies currently used for epileptic focus lateralization, such as magneto electroencephalography (MEG) and functional magnetic resonance imaging (fMRI) also depend largely on analysis of interictal spikes (Sakamoto et al. 2003; Al-Asmi et al. 2003).

\section{Material and methods}

This is a cross sectional study carried out in Electrophysiology Laboratory in Neurology Department of Dhaka Medical College Hospital (DMCH) from July 2010 to July 
2011. Our study population included 767 epilepsy patients. In the Lab room meticulous history and proper physical examination was done by neurologists. All the information was kept in record files. We selected only the cases with convincing history of seizure events, which were sent for EEG. They were advised to wash hair with shampoo and not to sleep overnight, so that patient can sleep on EEG table in the following morning. EEG was done within one month of seizure event. Each recording of EEG were obtained through digital equipments with minimal duration of 20-30 minutes and electrode positioned on scalp according to international 10-20 system. Recording was done in both awake and sleep state, except those who didn't sleep, only awakened state recording was taken. Provocative stimuli like hyperventilation, photic stimulation were given for three minutes each. For standardization, the background activity was classified as normal (organized and symmetrical) or abnormal (disorganized and/or asymmetrical). The EEG was interpretated by consultant neurologist, trained and experienced in electro-physiologic studies. The EEG was examined for specific epileptiform abnormality, the interictal spike or sharp wave. The abnormal electroencephalographic activity was also classified as generalized or focal. The presence and topography of bursts of slow waves and epileptiform paroxysms were evaluated. The latter were classified as spike-wave, sharpwave and polyspike. EEG was done within one month of last seizure and mostly after a single event. Though a total of 783 patients were initially interviewed, 16 patients with marked artifacts on EEG were excluded from this study.

\section{Results}

In our study, the patient's age ranged from birth to 64 years. The mean age at presentation to the EEG room was $9 \pm 2.36$ years i.e. most of the patients were less than 10 years old (39.9\%). The next common age group at seizure presentation was 11-20 years (33.2\%). Only 27 patients $(3.5 \%)$ were older than 50 years (Figure 1). Fifty seven percent of the patients were female and $43 \%$ were male (Figure 2). Out of 767 EEGs done in clinically diagnosed seizure patients, 294 (38.3\%) were normal and $473(62.7 \%)$ revealed abnormal. So the sensitivity of EEG in detecting electroencephalographic alteration in clinically suspected epileptic patients was $62.7 \%$ (about $69.6 \%$ below 20 years and $43.7 \%$ in those above 20 years). Among them $48.5 \%$ was diagnosed as localization related epilepsy (LRE) and $11.7 \%$ had generalized (GE) epileptiform discharge (Table 1). Among the patients with LRE, most common focus was temporal lobe (15.4\%) (Table 1). Regarding the morphology of epileptiform discharge, 74\% (341) had spike and wave, 11\% sharp and wave, $6 \%$ poly spikes and only $2 \%$ had slow waves (Table 2). Disorganized and or asymmetric background

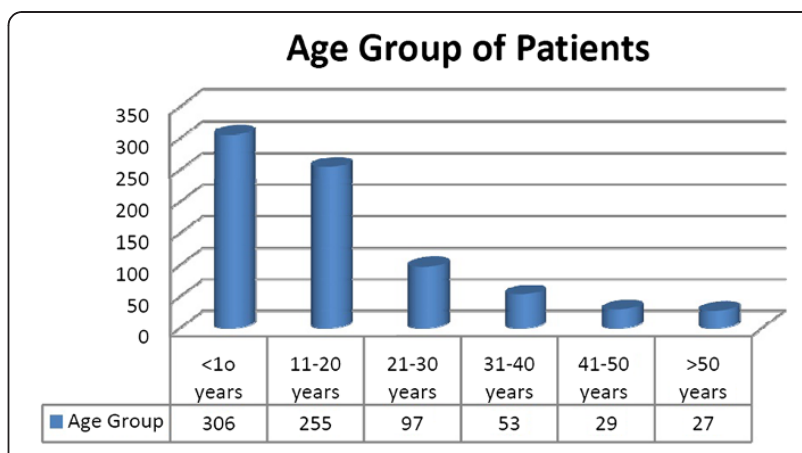

Figure 1 Age group of patients.

was recorded in $9 \%$ patients who had diagnosis of encephalopathy $(12,2.6 \%)$, mental retardation $(19,4 \%)$ and cerebral palsy $(11,2.4 \%)$.

\section{Discussion}

Epileptiform activity is quite specific for a diagnosis of epilepsy as the cause of transient loss of consciousness or other paroxysmal event which is clinically like to be epilepsy. In epileptic patients the EEG is examined for a specific epileptiform activity, the interictal spike or sharp wave. The spike or sharp wave represents the summated excitatory and inhibitory postsynaptic potentials associated with hyper-synchronous neuronal firing with paroxysmal depolarization shift and aftergoing hyperpolarization. This differing appearance, though not significant, reflects the rapidity of neuronal synchronization and the way in which the discharge spreads through the cortex. These discharges may either be focal or generalized. Since the beginning of use of EEG, it always has been a matter of query that how often the EEG does detect an interictal spike or sharp wave in an epileptic patient. Most of the patients in our study were children. This is probably due to the fact that parents are often frightened to observe a seizure event in their kid which led them to seek expert advice reasonably early. On the other hand adult patients are often reluctant to consult neurologists and often they prefer imaging first for clue to diagnosis of seizure etiology. Like our study, most of the reports regarding sensitivity of EEG are retrospective evaluation of data base from tertiary care centers. Several

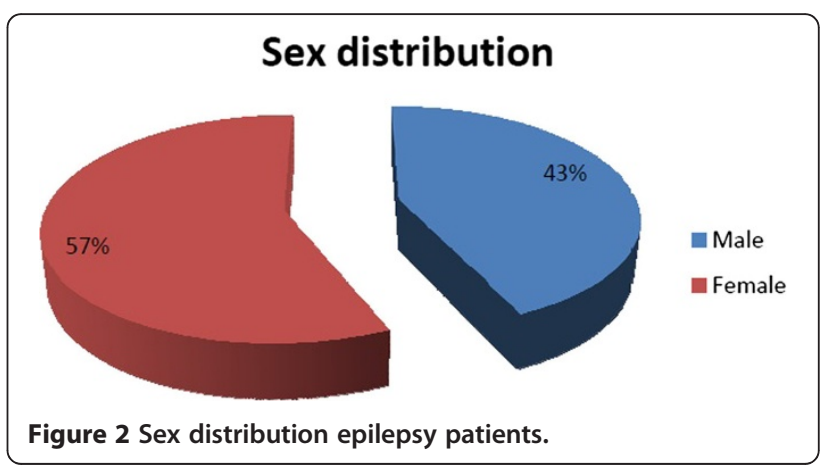


Table 1 Inter-ictal EEG of patients ( $N=767)$

\begin{tabular}{lll}
\hline EEG Findings & $\mathbf{n}$ & $\mathbf{\%}$ \\
\hline Normal & $\mathbf{2 9 4}$ & $\mathbf{3 8 . 3}$ \\
\hline Abnormal & $\mathbf{4 7 3}$ & $\mathbf{6 2 . 7}$ \\
\hline Localization Related Epilepsy & 372 & 48.5 \\
Frontal & 51 & 6.6 \\
Temporal & 118 & 15.4 \\
Parietal & 53 & 6.9 \\
Occipital & 19 & 2.4 \\
Central & 74 & 9.6 \\
Others & 57 & 7.4 \\
Generalized Epilepsy & 90 & 11.7 \\
Others & 11 & 1.5
\end{tabular}

Shows that out of 767 patients $62.7 \%(n-473)$ had abnormal EEG findings. Among these $48.5 \%(n-372)$ were LRE.

published studies on adult epilepsies showed that the chance of detecting interictal epileptiform discharges (IEDs) from the first EEG varies between 29\% and 55\% (Marsan \& Zivin 1970; Goodin et al. 1990; Salinsky et al. 1987) at outdoor monitoring of patients. Repeated EEG ultimately demonstrated the IEDs in $80 \%-90 \%$ of the patients (Marsan \& Zivin 1970; Salinsky et al. 1987). In video EEG monitoring also yields similar results. Walczak et al (1993) showed that long term video EEG monitoring can detect IEDs in up to $81 \%$ of the patients. Adoption of several methods can increase the chance of detecting IEDs. Sleep effectively improves detection of both generalized and focal IEDs (Angeleri 1975; Niedermeyer \& Rocca 1972; Sato et al. 1973). It is also likely to occur in an EEG if recorded early after a seizure event (Niedermeyer \& Rocca 1972). Hyperventilation and photic stimulation also induces IEDs in many patients, especially in generalized seizures (Sato et al. 1983; Miley \& Forster 1977; Wolf \& Goosses 1986). In healthy adult with no history of declared seizure, the incidence of epileptiform discharge is only $0.5 \%$. This also confers high sensitivity of EEG in detecting abnormal discharges (Gregory et al. 1993). Similar to other published reports, our study yielded abnormal EEG in most of the cases (62.7\%). Kershman et al (1951) reported in a series of

Table 2 Morphology of abnormal epileptiform discharge ( $N=473)$

\begin{tabular}{lll}
\hline EEG Findings & $\mathbf{n}$ & $\mathbf{\%}$ \\
\hline Abnormal background activity & $\mathbf{4 2}$ & $\mathbf{9}$ \\
\hline Spike and wave & 341 & 74 \\
Sharp and wave & 51 & 11 \\
Poly spikes & 29 & 6 \\
Slow waves & 10 & 2 \\
\hline
\end{tabular}

Shows that Spike and wave were the predominant epileptiform activity comprising $74 \%(n-341)$.
2648 patients with unquestionable diagnosis of seizure that $46.5 \%$ had focal changes while $15 \%$ had diffuse generalized abnormality in their scalp recordings of EEG. Our sample did not differ much in distribution of focal and generalized discharge (48.5\% and $11.7 \%$ respectively). In contrast to our finding, Dantas et al (2005) showed a lower sensitivity (43.6\%) of EEG, which probably accounts for the difference in age group of sample population. Betting et al. (2006) in his retrospective analysis of 493 EEG tracing from 180 generalized epilepsy patients showed that 33\% had generalized spike or polyspikes, while $22 \%$ had focal discharges in children. Morphologic analysis of EEG tracing in our study revealed a marked preponderance of spike wave paroxysms which comply with higher frequency of focal discharges.

We had some limitations. Firstly, there is chance of sampling bias. Moreover, the chance of inter observer biasness was minimized by following same principle in recording and typing the EEG abnormality.

\section{Conclusion}

The EEG is an important tool for diagnosing an epilepsy syndrome, knowledge of which aids in planning treatment and determining prognosis. Bangladesh being a third world country, has limited investigation facilities for epilepsy patients. Dhaka Medical College is running EEG lab. for a long time. And in Government level it is the only tertiary care hospital providing this facility. People from all sections and all corners of the country get the service from this hospital. So, the data presented here, gives us the actual scenario of EEG changes in patients with seizure disorder, for the first time. The presence of focal IEDs suggests the diagnosis of a localization related epilepsy, the character and location of which offer clues to both the etiology of the epilepsy syndrome and the location of the epileptogenic region. Finding of a generalized IED suggests the diagnosis of one of the generalized epilepsy syndromes. Thus the interictal EEG serves several purposes especially aids in detecting whether the epilepsy is present or not, helps in classifying the seizure disorder and also defining the epilepsy syndromes. With proper equipment and duration of recording through scalp electrodes it can detect abnormal cerebral neuronal discharges in two third cases of clinical seizure events. The finding of our study further consolidated the concept that EEG still remains the key investigation in clinically suspected epileptic patients and has a high level of sensitivity when performed in conditions properly indicated.

\section{Ethics}

The study protocol was approved by institutional ethical committee of Dhaka Medical College Hospital. 


\section{Competing interest}

The authors declare that they have no competing interest.

\section{Authors' contributions}

RN C was involved in planning, consultation, EEG reporting and data collection for this study. ATM HH was involved in data analysis and writing the manuscript. The rest except SRD were involved in EEG reporting and data collection. SRD was involved in data collection and planning of the study. All the authors have read and approved the final version of the manuscript.

\section{Data sharing}

There is no other unpublished data to share.

\section{Funding}

This research project was not funded by any group or any institution.

Received: 4 October 2012 Accepted: 23 January 2013

Published: 29 January 2013

\section{References}

Al-Asmi A, Bennar CG, Gross DW et al (2003) fMRI activation in continuous and spike-triggered EEG- fMRI studies of epilepsy spikes. Epilepsia 44:1328-39

Allen CMC, Lueck CJ, Dennis M (2010) Neurologic disease. In: Colledge NR, Walker BR, Ralston SH (eds) Davidson's Principles and Practice of Medicine, 21st edn. Elsevier Limited, p 1172

Angeleri F (1975) Partial epilepsies and nocturnal sleep. In: Levin P, Koella W (eds) Sleep. Karger, Basel, pp 196-203

Betting LE, Mory SB, Lopes-Cendes I et al (2006) EEG features in idiopathic generalized epilepsy: clues to diagnosis. Epilepsia 47(3):523-8

Dantas FG, Medeiros JLA, Nogueira BNF et al (2005) Papel do EEG em casos de suspeita ou diagnóstico de epilepsia. J Epilepsy Clin Neurophysiol 11(2):77-8

Gibbs FA, Davis H, Lennox WB (1935) The electroencephalogram in epilepsy and in conditions of impaired consciousness. Arch Neurol Psychiatry 34:1133-48

Goodin DS, Aminoff MJ, Laxer KD (1990) Detection of epileptiform activity by different noninvasive EEG methods in complex partial epilepsy. Ann Neurol 27:330-4

Gregory RP, Oates T, Merry RTG (1993) EEG epileptiform abnormalities in candidates for aircrew training. Electroencephalogr Clin Neurophysiol 86:75-7

Kershman J, Vasques J, Golstein S (1951) The incidence of focal and non-focal EEG abnormalities in clinical epilepsy. Electro encephal Clin Physiol 3:15-24

Marino R Jr, Cukiert A, Pinho E (1986) Aspectos epidemiológicos da epilepsia em São Paulo: um estudo de prevalência. Arq Neuro Psiquiatr 44(3):243-54

Marsan CA, Zivin LS (1970) Factors related to the occurrence of typical paroxysmal abnormalities in the EEG records of epileptic patients. Epilepsia 11:361-81

Miley CE, Forster FM (1977) Activation of partial complex seizures by hyperventilation. Arch Neurol 34:371-3

Niedermeyer E, Rocca U (1972) The diagnostic significance of sleep electroencephalograms in temporal lobe epilepsy. A comparison of scalp and depth tracings. Eur Neurol 7:119-29

Sakamoto S, Takami T, Hara M et al (2003) Interictal pattern of cerebral glucose metabolism, perfusion and magnetic field in mesial temporal lobe epilepsy. Epilepsia 44:1196-1206

Salinsky M, Kanter R, Dasheiff RM (1987) Effectiveness of multiple EEGs in supporting the diagnosis of epilepsy: an operational curve. Epilepsia 28:331-4

Sato S, Dreifuss FE, Penry JK (1973) The effect of sleep on spike-wave discharges in absence seizures. Neurology 23:1335-45

Sato S, Dreifuss FE, Penry JK et al (1983) Long-term follow-up of absence seizures. Neurology 33:1590-5

Walczak TS, Scheuer ML, Resor S et al (1993) Prevalence and features of epilepsy without interictal epileptiform discharges. Neurology 43(suppl):287-8

WHO report on Epilepsy in South East Asia (2999) Some facts and figures in Epilepsy, Avaialable at: http://www.searo.who.int/LinkFiles/Information_ and_Documents_facts

Wolf P, Goosses R (1986) Relation of photosensitivity to epileptic syndromes. J Neurol Neurosurg Psychiatry 49:1386-91

Yacubian EMT (2000) Epilepsia: o conceito atual. In: Yacubian EMT (ed) Epilepsia da Antiguidade ao Segundo Milênio. Lemos, São Paulo, pp 82-8

doi:10.1186/2193-1801-2-27

Cite this article as: Chowdhury et al:: Interictal EEG changes in patients with seizure disorder: experience in Bangladesh. SpringerPlus 2013 2:27.

\section{Submit your manuscript to a SpringerOpen ${ }^{\odot}$ journal and benefit from:}

- Convenient online submission

- Rigorous peer review

- Immediate publication on acceptance

- Open access: articles freely available online

- High visibility within the field

- Retaining the copyright to your article

Submit your next manuscript at springeropen.com 